\title{
Interoperable routing for IN and IP Telephony
}

\author{
Raimo Kantola*, Jose Costa Requena, Nicklas Beijar \\ Laboratory of Telecommunications Technology \\ Helsinki University of Technology, P.O. Box 3000, FIN-02015 HUT, Finland
}

\begin{abstract}
This paper discusses the problem of interoperability of IP Telephony and Switched Circuit Networks in the area of routing information. We describe and analyze a solution for directory-based routing. We will present an architecture based on a distributed database on the switched circuit network and the IP network. The aim of the solution is to provide efficient service management, close to optimal routing for all calls including calls to 800- and cellular numbers and personal number portability between both network technologies.

The solution is based on similar but differing in detail approaches for each network. In the IP network we use the Telephony Routing over IP (TRIP) protocol developed by IETF. Additionally, the described architecture is open for inclusion of the Telephone Number Mapping (ENUM) protocol that is under development. For the circuit switched network, we suggest a new protocol called Circuit Telephony Routing Information Protocol (CTRIP). The protocol provides a distributed method for synchronizing routing information between databases. Furthermore, we propose numbering gateways for transferring number information between the two network types. We utilize the Server Cache Synchronization Protocol (SCSP) as the common element for data synchronization and replication among distributed entities.

We also discuss briefly the design of a test implementation of the proposed infrastructure functions and protocols under development in our Laboratory. In conclusion, we propose to design a common interoperable routing information infrastructure for the emerging hybrid SCN/IP Telephony network.
\end{abstract}

Keywords: Voice over IP; number portability; directory; data replication; data synchronization; protocol; routing; SCSP; TRIP; CTRIP; ENUM

\section{INTRODUCTION}

IP telephony has been initially introduced in networks that are private from the point of view of the Switched Circuit Network (SCN). Today, a popular vision of the future network is that IP will carry all services and that the SCNs will finally be replaced by all services IP networks.

In this paper we will discuss the requirements that emerge in the area of management of routing information when we are moving from the phase of private IP Telephony networking towards the popular vision. We will also propose a solution to meet the requirements.

Since IP can not overtake SCN overnight, it is obvious that before we can achieve the popular goal of all IP networking, we must make the IP Telephony Network a full peer to the SCN. This means that the two technologies are connected using a public Network to Network Interface, such as ISDN User Part (ISUP) over IP. In addition, the IP Telephony Network and the SCN have more than one connection through gateways between each other. Because in both networks calls can be made from any extension to any other extension, in principle any gateway can be used to route any call, that needs to cross the technology boundary. We need automatic means to make an optimal or close to optimal choice of the gateway.

On the IP side the problem of gateway location for calls across the IP/SCN technology boundary was recognized a couple of years ago. Two Internet drafts have been issued by the IPTEL group of the IETF to establish clear requirements for the problem [4] and to provide a protocol based solution [5]. The proposed protocol, called TRIP, is used for both interoperator and intra-operator routing data distribution and synchronization between Location Servers (LS). The most recent version of TRIP is modeled on the Border Gateway Protocol (BGP-4). For data synchronization between multiple Location Servers in an administrative domain it uses the SCSP [1].

*Corresponding author.

E-mail addresses: raimo.kantola@hut.fi, jose@tct.hut.fi,nbeijar@tct.hut.fi 
The Signaling Servers can make queries to LSs on a call-by call basis to locate the most suitable gateway for a hybrid IP originated call. The current version of TRIP assigns the responsibility for number translations e.g. for 800-numbers to Redirect Servers which are a specific type of signaling servers present for example in the Session Initiation Protocol (SIP) architecture [6]. Alternatively, such redirection operations could be integrated into TRIP and the Location servers. This alternative seems especially attractive, if the LS query processing is stateless. The LS would send a subsequent query to another LS, SDP in an IN network e.g. for an 800 -number or to a Home Location Register (HLR) for a GSM -number.

The current TRIP specification leaves open the question of origination of the reachability data creating a considerable management burden in the future. This data essentially describes the SCN numbering space and maps it to gateways and gateway controllers suitable and willing to complete calls to the respective SCN number prefixes. For peer-to-peer connected SCN and IP Telephony Networks we are proposing to design a counterpart to TRIP which would distribute numbering information in the SCN e.g. between the IN Service Data Functions and across the technology boundary through Numbering and Routing Information Gateways (NRIGWs). The databases (SDFs) can reside in separate Service Data Points (SDP), be integrated in the Service Control Point (SCP), in adjucts or in switching systems. In our implementation we assume that IP -connectivity to these nodes is available. Due to the Circuit Telephony Routing Information Protocol (CTRIP), the information distributed by TRIP need not be manually entered into multiple LSs but is originated at its real source. This way the management of the numbering space can be largely policy based and the original information needs to be entered only once.

The rest of the paper is organized as follows. Section 2 elaborates on the motivation and defines the requirements for the interoperable routing system in a hybrid IP/SCN network. Section 3 gives a logical architecture that will meet the requirements. Section 4 describes the proposed new components in more detail. Section 5 animates the proposed architecture by showing a call setup example. Section 6 gives a brief description of our implementation of the proposed architecture and finally section 7 concludes.

\section{Motivation AND REQuiREMENTS}

The motivation for such an elaborate architecture is first the need to avoid unnecessary conversions on the media path between IP and SCN. The conversion causes delay and jitter and easily degrades voice quality to an unacceptable level. Therefore, in all possible cases optimal routing in terms of minimizing the number of conversions is desirable. An example in which an unnecessary media conversion could arise is a case of IP to SCN call when the B-number has ported out to an IP Telephony network and the originating IP network is not aware of this. Second, automation of service management in a multi-operator environment based on IP and circuit switched technologies is an economic necessity. The burden of this task to operators increases due to the dramatic increase in the number of IP Telephony operators. Number portability and roaming may move numbers inside an operator's network based on a single or based on the two technologies or even move numbers from one operator's network to another provided an arrangement exists such that billing can be reliably performed. Moreover, supporting number portability becomes attractive to operators in replacement scenarios where SCN technology is replaced by IP - obviously the replacement should not inconvenience users by requiring that they change their telephone numbers because of the change! Third, IP Telephone numbers can migrate in the IP telephony network topology and a different gateway may need to be selected by the SCN for an SCN originated call to complete a quality call to that number. We should also allow for originally IP phone numbers to be ported back to SCN. Consequently, the problem of gateway location is equally important for SCN originated calls as it is for the IP originated calls. Fourth, since the directory numbers in SCN are already mandated to be portable, description of the mapping of gateways to SCN numbering space needed for TRIP is a heavy management burden. Content of the TRIP information needs to originate somewhere.

Based on the discussion of the motivation we can formulate the essential requirements for the routing system as follows:

(1) Enough routing information should be available so that various routing criteria for hybrid IP/SCN calls become feasible: E.g. subject to end to end delay and policy constraints, select

- the least cost route through a gateway

- minimize gateway blocking probability (least loaded gateway)

(2) Routing is based on telephone numbers. Both DNS -style names (John.Smith@company.com) and telephone numbers can be used by computers, but the common denominator with SCN is telephone numbers. In particular, an IP phone that intends to be reachable from SCN shall have at least one directory number.

(3) Directory numbers are portable within geographical constraints irrespective of technology boundaries.

(4) The routing information needs to be available in the resources of the operators providing the service for a call irrespective of any changes in numbering or network technology. In particular, networks that have donated numbers from their original numbering space, should not be burdened with third party queries concerning those ported out numbers.

(5) All reachability information should be entered only once. The information should be made available to other operators automatically on a need-to-know basis. 
(6) Dynamic recovery from gateway faults and self-configuration to gateway capacity changes.

(7) Scalability in terms of size of the databases and number of operators. Scalability in terms of frequency of changes is a secondary concern.

\section{LOGICAL ARCHITECTURE}

\subsection{Information view}

Let us first define the key types of data that are used in routing calls in different networks. A Directory Number (DN) uniquely specifies a subscriber or a service. A geographically assigned DN can be directly used as a routing address. A non-geographically assigned DN, such as a service number or a ported out directory number, needs to be mapped into a routing address before routing can be performed.

A Routing address points to an extension in a network. By aligning the allocation of routing addresses with network topology, prefixes of routing addresses are enough for routing in most of the network. A Routing number is a routing address used in SCN and follows the E.164 format.

A portable number is a DN with a number prefix such that at least one ported out number with that prefix exists. The prefix is of the length typically analyzed by the exchanges or signaling servers in the network without the help of external databases. A ported out number is a DN that is attached to a telephony extension in an exchange or network different from the exchange or network it originally belonged to.

Number mapping from directory numbers to routing addresses has a central role in the infrastructure. If the destination is on an SCN, the directory number is mapped into a routing number using directories. Correspondingly, if the destination is on an IP-network, the directory number is mapped into an IP-address of either the terminal device or a signaling server serving the terminal.

The mapping function is divided into two parts. First, the directory number is mapped into a destination network. With the destination network we refer to the collection of resources based on one technology (IP or SCN) belonging to one operator. Second, the directory number is mapped into a routing address that is a routing number on the SCN and an IP-address on the IP-network. The address is only valid within the destination network.

At a minimum, there should be enough information available to obtain the destination network type and operator using the directory number as the key. To perform the mapping function, we need at least the following information for each number:

- Directory number (key field).

- Destination operator.

- Destination network type (SCN, IP).

- Destination routing address. Format depends on the destination network type (E.164, IP-address).

\subsection{Current Number Portability solution based on IN}

The current Number Portability architecture (see Figure 1) in SCN in most western European countries and North America is based on translating the portable numbers using the Service Switching (SSF), the Service Control (SCF) and the Service Data Functions (SDF) of the originating or some transit operator. The SDF returns a routing number for a ported out number and the original directory number if it has not been ported out. For the query to the IN nodes, different protocols are used in different networks and by different operators. Examples are IN Application Part (INAP) and Mobile Application Part (MAP).

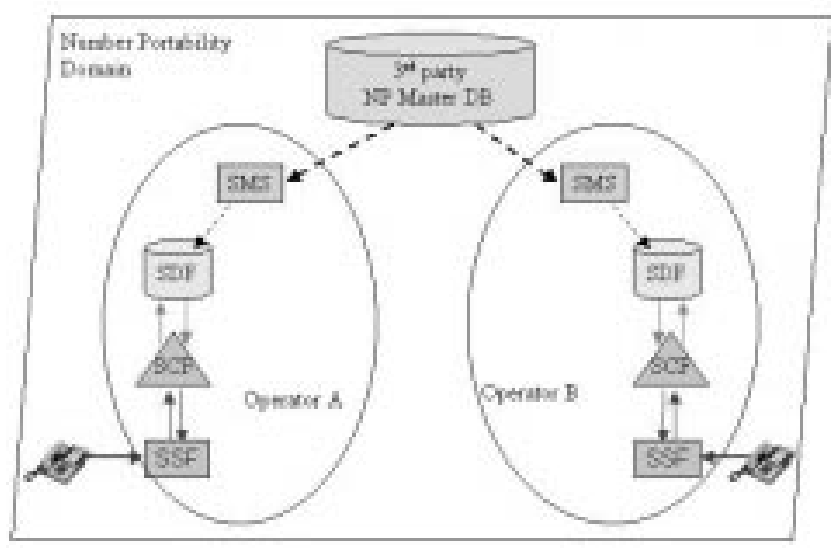

Figure 1. Current NP Architecture based on IN 
The management of the ported numbers is typically done using a third party master database, which has records of all ported out and portable numbers. The third party might be e.g. an independent company mandated by the regulator to carry out the function or a joint organization by all the operators in the area in which number portability is supported. The master database will download its contents to the Service Management Systems (SMS) of all the operators in the area. The SMS in turn will manage the contents of the SDF. There is no global standard for the interface between the master database and the local SMS. The implementations vary from country to country.

If porting numbers has not been made economically unattractive to the customers, this architecture leads to a situation in which all DNs must be considered portable and two queries to the IN per each call in the network has to be made. One query to translate the B-number and another to translate the A-number, which also needs to be carried in call signaling. At the same time, this architecture does not support management of number portability by the users themselves.

\subsection{Protocols}

In our architecture we would like to utilize the existing protocols as much as possible. The protocols under consideration are TRIP and ENUM for location of gateways and terminals in the IP network, and SCSP for synchronization and replication of distributed databases. We would also like to utilize the framework for IN-based number portability in the SCN. However, instead of using a master database for a number portability area, we prefer a distributed solution where the information is located in the nodes where it is needed and the updates are performed by the entities involved in the transaction of porting a number.

On the IP network TRIP distributes information about the gateways, which connect the SCN and the IP-network, and about the number ranges reachable through these gateways. TRIP could also be used to distribute information about IP terminals, but the current plans define a separate system for that: ENUM. ENUM utilizes the existing Domain Name Service (DNS) to distribute information about the terminals residing on the IP-network. Together TRIP and ENUM cover all terminals reachable from the IP-network.

On the SCN, numbering information is used by the nodes of the Intelligent Network. The information is stored in databases in the Service Data Functions (SDF). Our goal is to develop a completely distributed solution. A protocol is required to distribute and synchronize information between the databases. We develop a protocol based on SCSP to perform database replication and synchronization. Since the resulting protocol will be used to distribute routing information over the switched circuit network, we call it Circuit Telephony Routing Information Protocol (CTRIP). CTRIP carries numbering information about both SCN- and IP-terminals.

\subsection{Protocol interaction}

The information about E.164 numbers in both network types must be consistent. This requires interaction between the protocols and translation of information. An automatic system for the updates is required, since updating the databases separately for every change would be an unnecessary economical burden and would result in inconsistency in the end. We need an automatic converter to transfer the information from protocol to protocol when numbering information changes in one network. We will call this entity a Numbering Gateway. Three types of conversions may be needed:

1. Update from CTRIP to TRIP

2. Update from ENUM to CTRIP

3. Update from TRIP to CTRIP

The situation is illustrated in Figure 2.

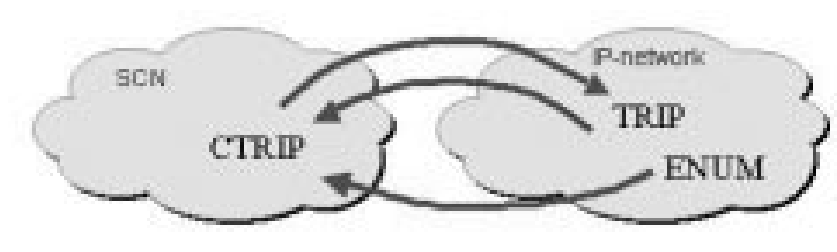

Figure 2. Interaction between CTRIP, TRIP and ENUM

\subsection{Network architecture}

The architecture consists of SCN networks and IP-networks owned by different operators. We use the term network technology to differentiate between the SCN and IP technology. The term network refers to the network of one operator based on one network technology. Each network runs at least CTRIP or TRIP, depending on the network technology. 
Additionally the IP network can use ENUM. On the IP side, the resources belonging to the same administrative authority (operator) constitute an IP Telephony Administrative Domain (ITAD). The reference architecture is depicted in Figure 3.

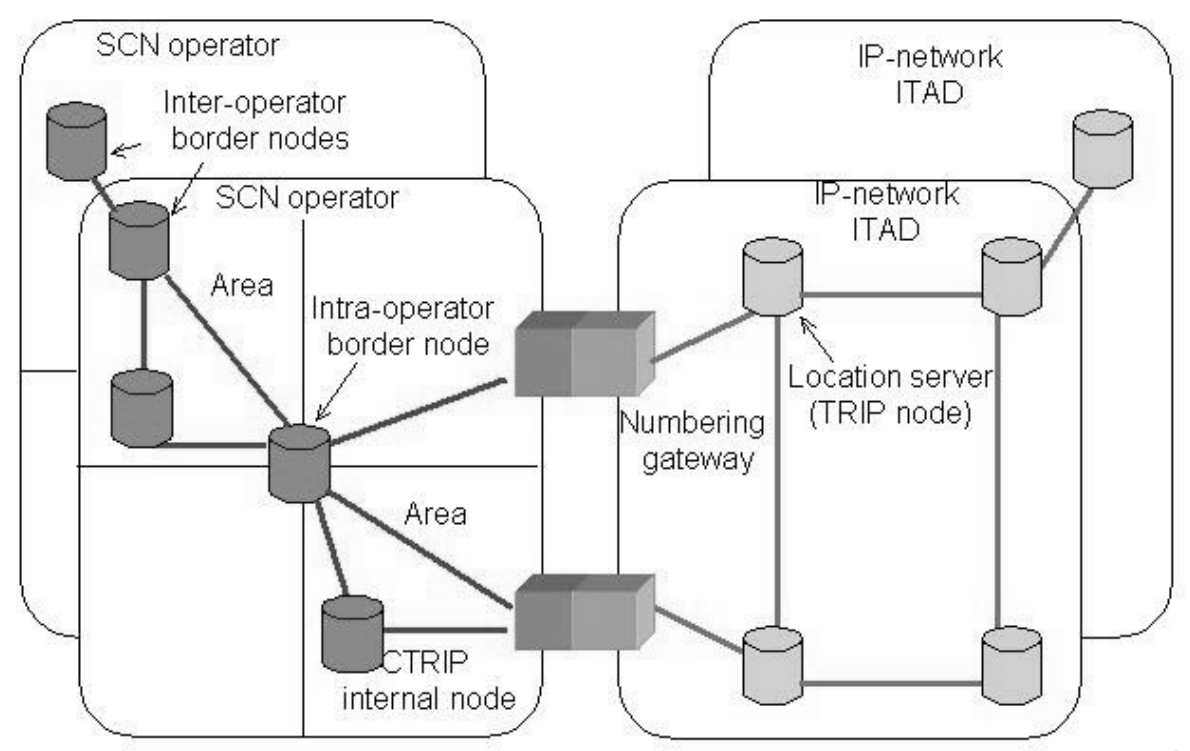

Figure 3. Reference architecture

\subsubsection{The network functions}

On the switched circuit network, the numbering databases are accessed by the Service Control Functions (SCF). We assume in our implementation that the database has IP connectivity. Alternatively, SCSP could be modified to run over the Signaling Connection Control Part (SCCP) of the Signaling System Number 7.

On the IP network, numbering information is stored in the Location Servers (LS). A DNS server is one type of Location Server. As calls are being set up, the call setup passes through one or several Signaling Servers (SS), which query numbering information from the location servers. In this work, we will use the term signaling server also for the gatekeeper defined in the H.323 recommendation. In implementations the location server functionality can be integrated with the signaling server. If they are not integrated, the signaling server uses a directory access protocol such as the Lightweight Directory Access Protocol (LDAP) or DNS lookups for accessing information in the location servers.

\subsubsection{Numbering space model}

Within the SCN we will use CTRIP over SCSP to distribute information between numbering databases. SCSP sets up peerto-peer relationships between CTRIP nodes. The information is synchronized between all nodes in a server group, so that all the databases will contain the same information. With CTRIP we distribute the information within an operator's network. We further divide the network into areas that can be defined by the operator. The areas constitute separate server groups, so all the CTRIP nodes within an area contain the same numbering information. To distribute the information between areas and operators, some nodes belong to two areas. These are called border nodes. Depending on the type of the border, they are either intra-operator border nodes or inter-operator border nodes. The border nodes belong to two server groups and they transfer information between the two groups. Aggregation and policy rules can be applied in the transfer.

TRIP uses the Border Gateway Protocol model to distribute routing information between ITADs. Policies and aggregation can be applied on the borders between the domains. Within an ITAD all databases contain the same information. TRIP uses SCSP to synchronize the databases within an ITAD.

ENUM uses the existing Domain Name Service (DNS) to store IP phone numbers. Lookups are distributed in hierarchical order from a root server to more specific servers.

\subsubsection{Numbering gateways}

As defined in section 3.3, we have three cases when information may need to be transmitted between the network types. The information about SCN-terminals is translated from CTRIP to TRIP and the attributes of the gateway are attached. In the opposite direction, the information about IP-terminals is translated from TRIP to CTRIP or ENUM to CTRIP. Since these are quite independent functions, they can be implemented as separate gateways, at least logically. We will call them 
CTRIP-to-TRIP gateway, TRIP-to-CTRIP gateway and ENUM-to-CTRIP gateway respectively. In a situation where they are implemented as a single gateway, the terms refer to the corresponding logical functionality. Although each gateway role involves only two protocols, the gateways need connectivity to the third protocol in order to be able to remove previous entities with the same key.

The CTRIP-to-TRIP gateway also has to be able to retrieve information about the media gateway, since the TRIP protocol advertises the gateway properties in addition to the number ranges reachable through it. One could use some protocol to access the properties directly from the gateway. This has some significant drawbacks. In our model, we will expect that the gateway information is available in the location servers. The gateway will thus register its properties to one or several location servers. The numbering gateway queries the location server to learn about the gateways it should advertise. It obtains the addresses of the media gateways and their attributes. The exact protocol to be used for this purpose is not defined, but a good candidate is LDAP.

\subsection{Aggregation and policy}

To reduce the amount of information stored in the databases and transported over the network, aggregation can be used. The idea of aggregation is to represent a range of several numbers as a single entry. Aggregation is possible for ranges of numbers with the same properties. One of the properties that have to be equal is the destination. A consequence of this is that entries representing end terminals can not be aggregated. However, a group of terminals on the same destination network can still be aggregated outside their network. The group can be represented as a single entry if they have a common prefix and the other properties are equal. Aggregation requires that the destination networks know the more detailed addresses. The source network can thus route the call to the correct destination network and the destination network locates the terminal using a more detailed routing address. Under these conditions, aggregation is possible between different operators and network types.

Aggregation is also possible within a single operator's network if the network is divided into areas. Each area acts like an independent network from the viewpoint of routing. A range of terminals in the same area is represented as a single entry with the same destination region on other areas. Another advantage of dividing the network into areas is that the use of network resources can be optimized. When a call is set up between two operator's networks, the call setup can immediately be directed to the correct area of the end terminal.

SCSP requires the information to be equal within a server group. The division of the CTRIP routing domain into areas makes the server groups smaller. All numbering databases inside an area must contain the same information. Each area must have the exact routing addresses of the end terminals within the area. Information about terminals in other areas and other operator's network can be aggregated. The aggregation is performed by the border nodes, which belong to two areas or networks. Information is aggregated before it is transmitted to the neighboring network or area.

Correspondingly, there are border nodes at the borders between operators. In addition to the aggregation, these nodes can apply policy rules on the information transferred to the neighboring operator. They can remove unnecessarily exact routing information such as the destination routing numbers. Only the destination network information is required for establishing calls successfully. Possibly also the area information can be removed, but this will make routing more inefficient. The principle of policy in this case, is that the network decides what information is given out to the neighboring network. For cases where two networks have many connections we may need to define some policy attributes that need to be sent to the neighboring network along with the reachability information. Aggregation and policy are applied at the node where information is sent to the neighboring network, but further aggregation can be done at the receiving node.

\section{NEW COMPONENTS}

In this section, we will discuss the information and the mechanisms required on the SCN-side. We will also describe the numbering gateway functions that connect the SCN and IP networks and create a seamlessly interoperable routing system. We use existing TRIP attributes without modifications.

\subsection{Required fields in CTRIP}

Since the planned architecture builds on a similar call establishment procedure as that of IN-based number portability, we can expect that the same fields are required in CTRIP. The fields proposed for IN-based number portability are the following:

- The directory number of the subscriber (key field)

- The destination operator serving the subscriber

- The number portability routing domain, where the subscriber is located.

In addition we will need at least the following fields:

- Routing number

- Signaling capability identifier

- Area 
- Advertisement path

- State

To allow for whole ranges of numbers to be portable, we will use a directory number prefix instead of single numbers. As with IN-based number portability, we need to know the operator of the subscriber in order to locate the destination network.

We include a field for the routing number. The field is used to make the second phase mapping in the destination network. The use of this field outside the destination network is subject to a policy decision. For distribution policy control of the routing number, we use the number portability routing domain. However, this is a policy attribute and does not need to be passed around by the protocol.

The Destination Operator and Destination Network fields are often used together to indicate the destination network where the call is routed. They can be combined to form a Sub-domain Identifier (SDI), which uniquely identifies the combination of network and operator within the routing domain. It thus replaces the operator identifier and allows a distinct indication of IP- and SCN networks, which is necessary in order to include IP networks in the IN-based model.

State information is required to avoid routing loops and to coordinate updates between the nodes. The state information is related to the entry, and each entry has a state that is independent of other entries. Now we examine this field in detail.

\subsection{State information requirements}

Without state information, it is possible for the following situations to occur:

- An operator allocates a number that is in use by another operator.

- An operator deletes a number that is in use by another operator

- A number is mapped to a different terminal in CTRIP and in the external protocol (e.g. TRIP).

- A number is mapped to an SCN-terminal on the IP-network and to an IP-terminal on the SCN.

These problems can be avoided by maintaining state information and using it to indicate whether the number is free, allocated, moving or unallocated. A free number is available for allocation to a subscriber. An allocated number is in use by a subscriber. A moving number is not in use but it is still reserved to a subscriber for reallocation in a new operator's network. An unallocated number is not related to any subscriber. It is not in use, but can not either be allocated to another subscriber within a specified period. Calls can only be established to an allocated number.

Since we also have entries originating from external protocols, like TRIP and ENUM, we also need to separate between internal and external entries. External entries are created by the numbering gateways based on the information that the external protocol provides. These should not be handled in the same way as internal entries. In order to prevent loops, they must not be announced back to the external protocol. Detailed protocol interactions related to these numbers may also differ from native SCN number interactions. Gateways might also be required to regularly update the external entries as an indication that they still exist in the external protocol. External entries can have similar allocation information (free, allocated, moving, unallocated) as internal entries, but only the allocated state is specific to external entries. The free, moving and unallocated states are not specifically related to internal or external protocols. We also want to indicate whether the number is available or for some reason unavailable.

It is possible to have separate fields for allocation, originating protocol and availability. However, since all information affects the state of the entry and the possible state transitions, we prefer to use a single state variable.

The advertisement path attribute is used to eliminate loops in the distribution of the information.

\subsection{Numbering gateways and external entries}

The numbering gateway is the entity transferring information about telephone numbers between different protocols. 


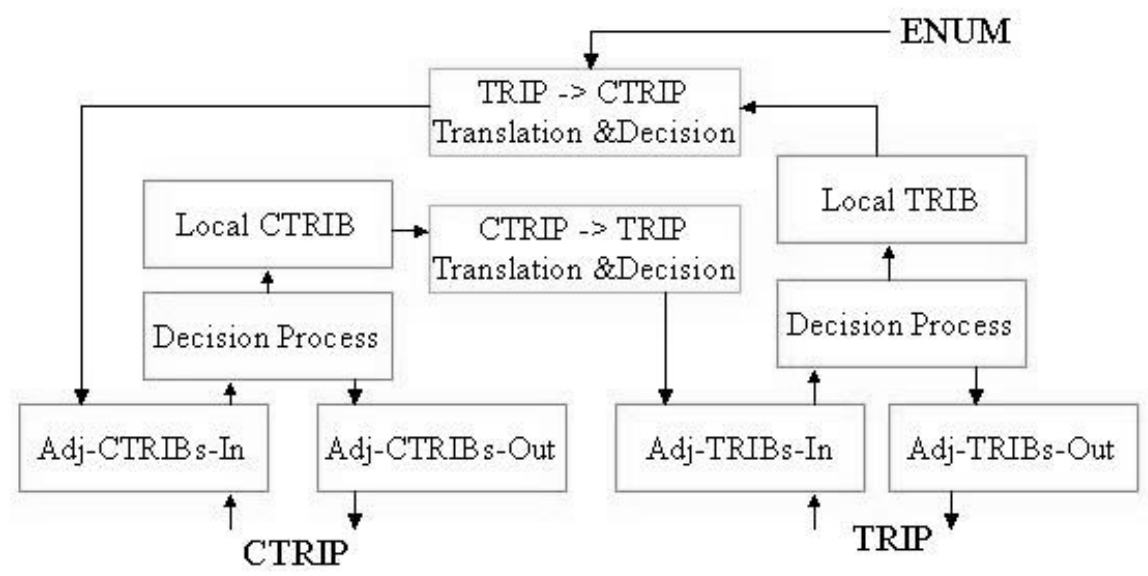

Figure 4. Structure of a numbering gateway

A general structure of the numbering gateway is shown in Figure 4. The numbering gateway is a combination of a TRIP and a CTRIP node. Between the nodes there are translation functions that transfer information between the two databases.

We have described the functionality of the new components in more detail in [10].

\section{CALL SETUP PROCEDURE}

We will show call setup using an example where a call is set up from SCN to an IP network.

In the model, we separate between the logical layers used for call setup and distribution of call routing information. This is illustrated in Figure 5. The entities in the call setup layer are involved in call signaling. The layer above is used for distributing of routing information using CTRIP, TRIP and ENUM. Information flow between the layers is performed using directory access protocols such as LDAP and DNS lookups on the IP network and INAP on the SCN.

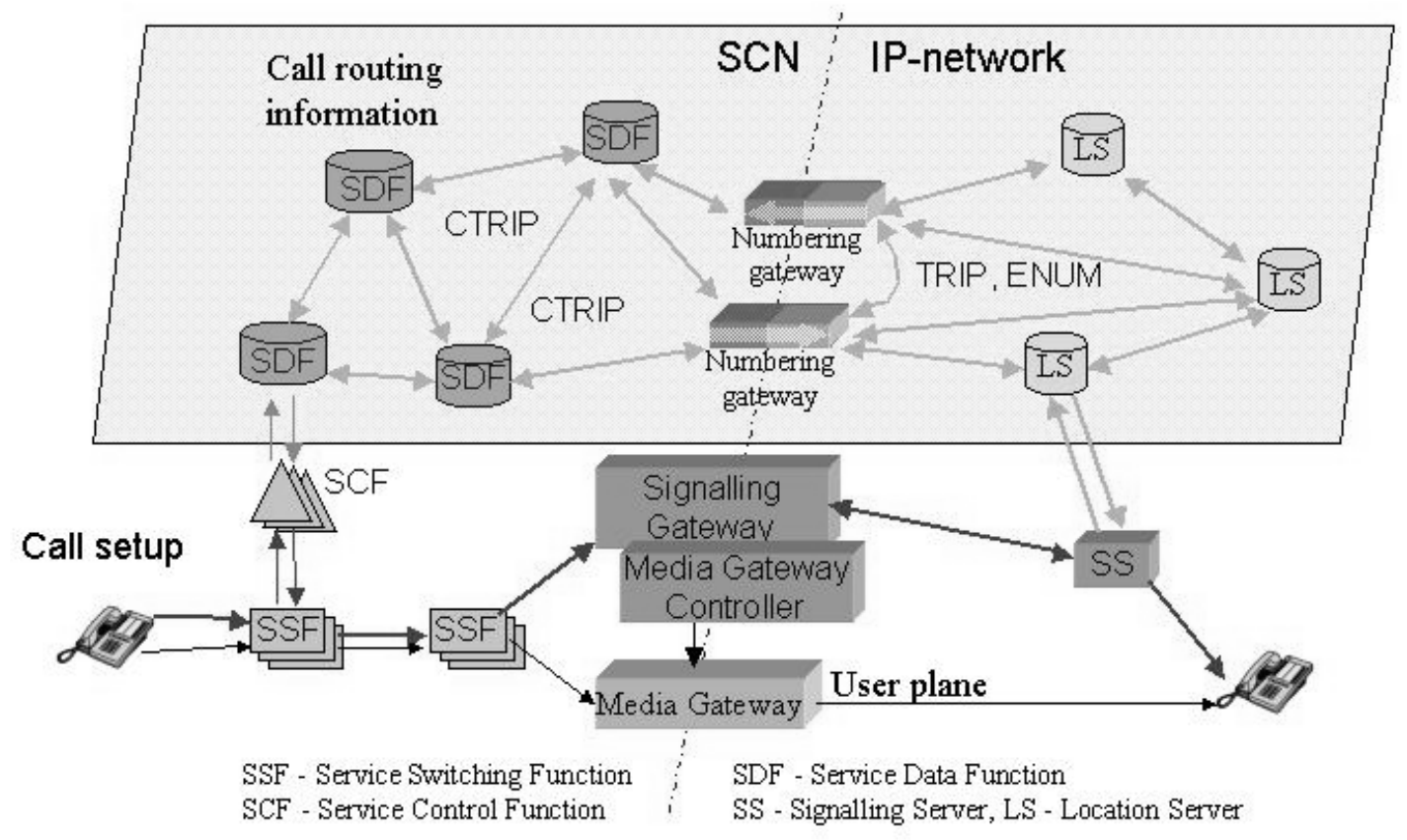

Figure 5. Call signaling and call routing information layers 
The call setup information flow in our call case is shown in Figure 6. The numbering databases are accessed in the setup phase of a call. Our presentation will skip a lot of the details because a lot of variants are possible within the framework and a lot of the details are still under consideration in standardization bodies.

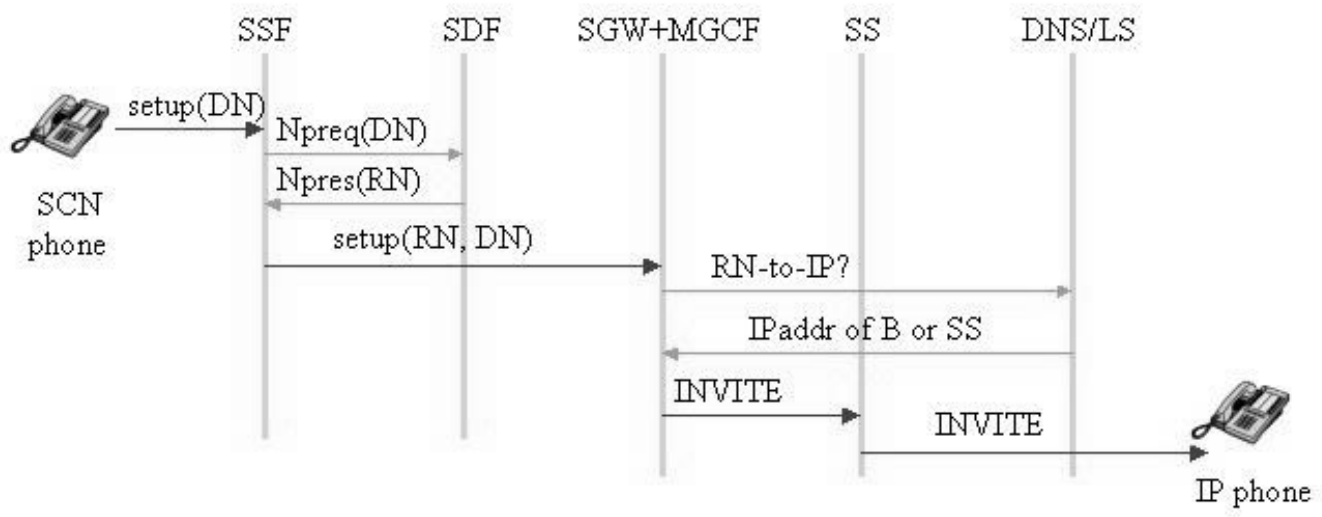

Figure 6. Call example Information flow

The subscriber dials the directory number of the destination. A suitable SSF capable exchange performs the IN query. This takes place in the originating network or in the transit network. The numbering database in the SDF is accessed, and the result of the query is a routing number pointing towards the destination network. The concerned SDF entry is automatically managed by the CTRIP and the SMS on the background.

The format of the routing number depends on the type and location of the destination. In addition to the routing address of the terminal, the routing number also includes information about the area, network type and operator of the destination.

The routing tables in the exchanges of the originating and/or the transit network are set to route these calls to an appropriate gateway to the correct IP Telephony area. Preferably the area that created the CTRIP entry is used.

Outgoing signaling from the SSF that performed the query must also carry information that the location query has already been made so that no other node on the call path will want to repeat the query. For translating the signaling from an SCN signaling system to an IP Telephony signaling system the SCN may use a signaling gateway and a media gateway controller. The latter also controls the appropriate media gateway which transcodes the voice path between the two technologies. When the call reaches the signaling gateway, it initiates an IP call setup procedure on the IP network. In the IP call the signaling and the media paths are separate. The signaling servers are responsible for the call setup. In our case, the Session Initiation Protocol (SIP) is used for call signaling. The signaling servers on the call path utilize location servers to locate the next hop signaling server and most likely the DNS to locate the destination. The lookup is performed using some directory access protocol, for example LDAP. The information in the location server concerning an IP destination originates from ENUM or possibly TRIP.

The call passes through a number of signaling servers on its way to the destination. These either redirect or proxy the setup to the next step server. In Figure 6 proxy signaling is used. The last hop is the terminal itself or the home server of the terminal. Suppose that the IP - user is available and will want to communicate (omitted in Figure 6). Answer is signaled back to the signaling gateway, which with the help of the media gateway controller will set up the media gateway into transcoding the bi-directional media path. The signaling gateway will also pass the answer signal back towards the originating exchange so that charging can be initiated, the two way call path established and the talk phase can begin.

\section{IMPLEMENTATION}

The structure of our implementation is depicted in Figure 7. The SCSP protocol [3,7] supports loose data synchronization in a server group of an arbitrary topology. The SCSP is based on the proven link state interior packet routing protocol for the Internet called the Open Shortest Path First, OSPF -protocol. The SCSP has three sub-protocols. First, the servers need to establish neighbor relationships, second they need to accomplish initial data synchronization by exchanging complete copies of their databases. In normal operation the SCSP servers will flood the changes in their data to their neighbors in the server group as the changes appear in the originating server. The SCSP has limited means for implementing elaborate data distribution policies; therefore, TRIP uses both the SCSP and the BGP-4 models. 


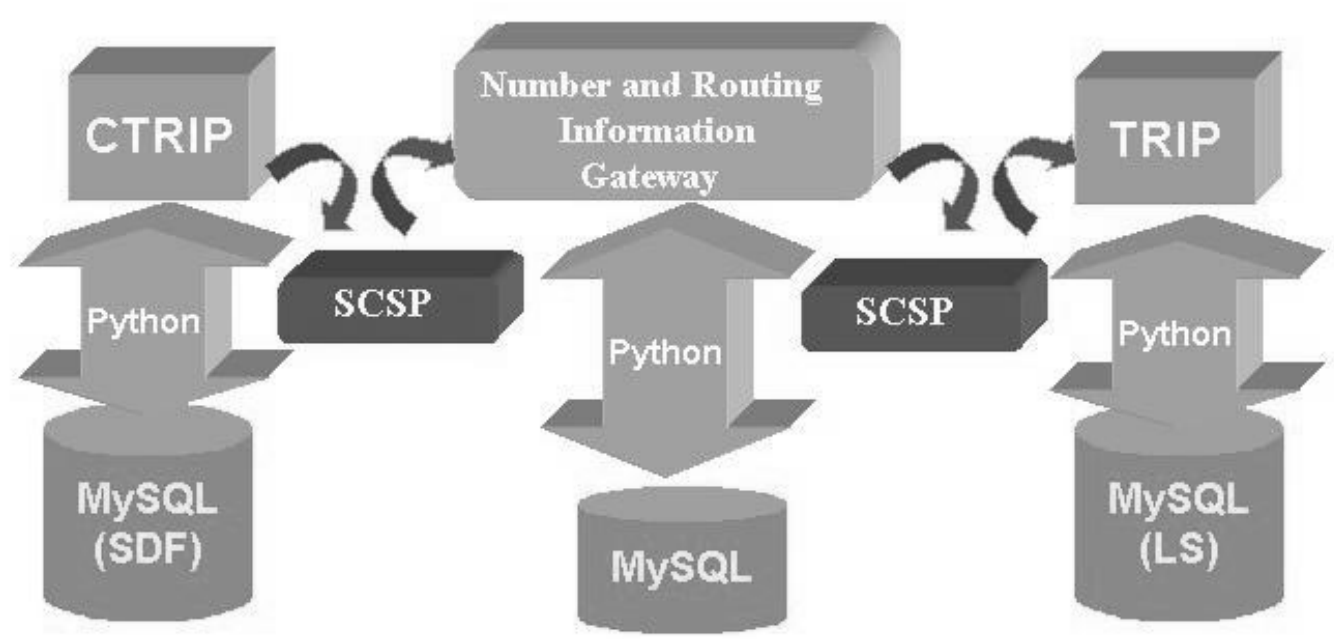

Figure 7. The architecture of the Numbering and Routing Information testbed

We have chosen MySQL as the routing information database in order to bring the power of relational database tools to our environment and because MySQL is a public domain package [8]. At the moment, for flooding of the routing information between SCN and IP we use the SCSP protocol, which was implemented in C++ in our project [3, 7]. The algorithms and data management specified by the TRIP protocol and its circuit counterpart has been developed using Python [2] (see Figure 5).

Information about the IP-telephones themselves may end up being stored in the DNS, as being studied in the IETF ENUM working group. We have discussed our implementation in more detail in [9].

\section{Conclusions}

A novel and comprehensive approach for numbering interoperability between SCN and IP has been proposed. It is easy to see that the solution meets requirements (2)...(6) of section 2. For the purpose of meeting all the routing criteria requirements new routing attributes may need to be added to our system. The project for implementing a comprehensive routing information testbed is under way for the purpose of evaluating new telephony routing features and studying the scalability of proposed solutions. A design goal of the testbed is the ease of adding new routing attributes. We propose to extend the model of distributing routing information among Location Servers adopted on the IP Telephony side by the IETF also to the SCN side. We believe this is the way to achieve seamless interoperability between the two networking technologies. We believe that this is a necessary step in order to automate service management while we are moving towards all IP Networking where all communications services run over the ubiquitous IP network. To make this happen, standardization of the described CTRIP protocol should be undertaken by the appropriate standardization bodies.

The proposed solution is service management centered. It also facilitates self-management of number portability by the subscribers themselves although due to lack of space, we have not shown this in detail. In this respect it potentially reaches much farther than the current number portability management solutions based on classical IN adopted in North America and in Europe. Our proposed solution will require changes to the service management in IN networks. Third party master databases of the ported and portable numbers are no longer needed. When a number is ported from one operator to another, the SMSs of the two operators are involved and will authorize the state changes of the number entries. We believe that these changes are justified due to the lower cost of service management and added convenience to the end customers.

\section{Acronyms}

BGP-4 - Border Gateway Protocol version 4 (RFC 1771)

CTRIP - Circuit Telephony Routing Information Protocol

DNS - Domain Name Service

GSM - Global System for Mobile Communications

ENUM - Telephone Number Mapping

E.164 - ITU-T specified telephone number format

HLR - Home Location Register

IETF - Internet Engineering Task Force

IN - Intelligent Network 
INAP - IN Application Part

ISUP - ISDN User Part

ITAD - IP Telephony Administrative Domain

LDAP - Lightweight Directory Access Protocol

LS - Location Server

MAP - Mobile Application Part

OSPF - Open Shortest Path First

SCCP - Signaling Connection Control Part

SCN - Switched Circuit Network

SCSP - Server Cache Synchronization Protocol

SIP - Session Initiation Protocol

$\mathrm{SCF}$ - Service Control Function

SCP - Service Control Point

SDF - Service Data Function

SDP - Service Data Point

SS - Signaling Server

SSF - Service Switching Function

SMS - Service Management System

TRIP - Telephony Routing Information Protocol

\section{REFERENCES}

[1] J. Luciani, G. Armitage, J. Hlapern N. Doraswamy. "RFC 2334. Server Cache Synchronization Protocol" . Network Working Group. Standards Track, April 1998.

[2] A. Watters, G. van Rossum, J. Ahlstrom. "Internet Programming with Python". M\&T Books 96.

[3] J. Costa Requena, "An Implementation of the Server Cache Synchronization Protocol, M.Sc thesis, Laboratory of Telecommunications Technology, Helsinki University of Technology, 1999.

[4] J. Rosenberg, H. Schulzrinne, A Framework for a Gateway Location Protocol, IETF Internet Draft, draft-ietf-iptel-gwlocframework-03.txt, work in progress, June 1999.

[5] J. Rosenberg, H.Salama, M.Squire Telephony Routing over IP (TRIP), IETF Internet Draft, draft-ietf-iptel-trip-01.txt, Work in Progress, January 2000.

[6] M. Handlay, H. Schulzrinne, E. Schooler and J. Rosenberg, SIP: Session Initiation Protocol," IETF Internet Draft, draft-ietfmmusic-sip-12.txt, Work in Progress, January 1999.

[7] Jose Costa Requena, Raimo Kantola, "Server Cache Synchronization Protocol (SCSP): component for directory enabled networks", SPIE Photonics, Boston September 1999

[8] http://www.hughes.com.au/products/msql/

[9] Raimo Kantola, Jose M. Costa Requena, and Nicklas Beijar, "An Architecture for an SCN/IP Telephony Routing Testbed", the $1^{\text {st }}$ IP Telephony Workshop, Berlin, April 2000.

[10] Raimo Kantola, Jose M. Costa Requena, and Nicklas Beijar, "A Common Numbering Infrastructure for IN and IP Telephony, IN2000 workshop, South Africa, May 2000.

\section{Biographies}

Raimo Kantola graduated with an M.Sc. in Computer Science in Leningrad Electrotechnical Institute in 1981. He received the Doctor of Technology degree in Computer Science from Helsinki University of Technology in 1995. Dr. Kantola worked for Nokia for more than 15 years on Switching R\&D and Product marketing and joined HUT as a professor of Telecommunications Technology in 1996.

Jose Costa Requena graduated with M.Sc. in Engineering in Polytechnic University of Valencia in 1999 having done his Master's project at HUT. Jose joined HUT to work on SCN/IP telephony Interoperability architecture in 1998. From 1999 Jose is a Ph.D student at the Laboratory of Telecommunications Technology leading a group of M.Sc. students on the routing testbed project.

Nicklas Beijar is an M.Sc student and joined the Laboratory of Telecommunications Technology in 1998 as a research assistant. Nicklas is now finalizing his M.Sc. thesis on CTRIP. 